\title{
Carbohydrate Metabolism Pathway
}

National Cancer Institute

\section{Source}

National Cancer Institute. Carbohydrate Metabolism Pathway. NCI Thesaurus. Code C39726.

An elaboration of a sequence of biological or biochemical events that play a role in the metabolism of carbohydrates. 